\title{
The holy grail for plant biologists
}

Plant biologists and biotechnologists have long suffered from the lack of an efficient and sequence-specific method for gene targeting. Two recent reports of successful gene targeting in maize and tobacco come as a welcome improvement on the laborious conventional mutagenesis or transgenesis approaches. For the first time, endogenous plant loci can be targeted at high efficiency.

Both studies rely on zinc-finger nucleases (ZFNs) - engineered enzymes that create double-stranded breaks at specific loci and that have

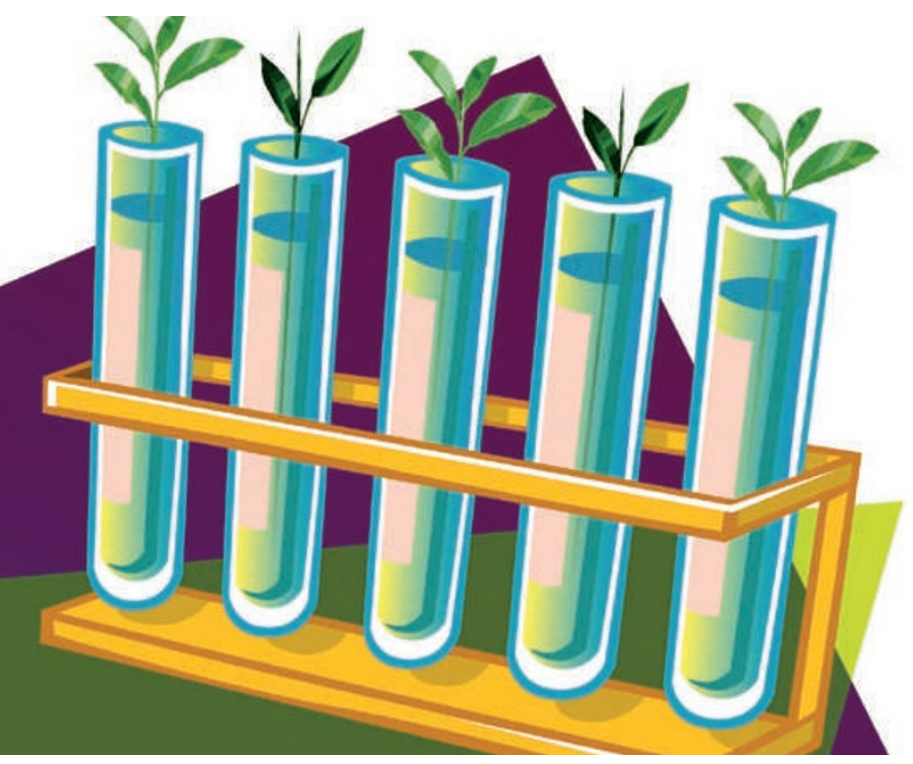

previously been used to modify, in vitro, plant transgenes and endogenous genes in human cells. ZFNs are a fusion between an endonuclease domain and a zinc-finger-based DNA recognition domain; this latter domain can be designed to recognize almost any DNA sequence, and therefore gives ZFNs their specificity.

Working in maize, Shukla and colleagues used a panel of prevalidated ZFNs that were designed against two independent endogenous loci: IPK1, the product of which catalyzes the final step in phytate biosynthesis in seeds, and ZP15, which encodes a seed protein. Following pre-screening to determine the relative efficiency of their ZFNs, the authors detected modification in plants by selecting for the insertion of a herbicide tolerance gene or by amplifying and sequencing the targeted locus. As transgene insertion is very specific the authors were able to generate several independent lines of fertile plants that transmitted the modification to the next generation.

In an independent effort, Townsend and Wright et al. report the modification of multiple acetolactate synthase loci in tobacco plants; when inactivated, these genes render a plant resistant to two types of herbicide. For their ZFN design they relied on a publicly available resource from the Zinc Finger Consortium, and selected the molecules with highest specificity by pre-screening them in bacteria and subsequently in yeast. Interestingly, in a small proportion of cases they also saw targeting over $1.3 \mathrm{~kb}$ from the site of cleavage. This suggests that it should be possible to modify plant genes even if their surrounding genomic sequence is not optimal for ZFN targeting.

The precision and efficiency of the ZFNs offer clear advantages for dissecting gene function in plants as well as for plant engineering for food or fuel. Given the versatility of ZFNs, this approach can be used in any plant species as long as it is amenable to DNA delivery. In the future, targeted modifications could be identified in DNA-sequence-based screens; considering the advances in sequencing technologies, high-throughput plant genomic engineering could be just around the corner.

Magdalena Skipper, Senior Editor, Nature

ORIGINAL RESEARCH PAPERS Shukla, V. K. et al. Precise genome modification in the crop species of Zea mays using zinc-finger nucleases. Nature 28 Apr 2009 (doi:10.1038/nature07992)| Townsend, J. A. \& Wright, D. A. et al. Highfrequency modification of plant genes using engineered zinc-finger nucleases. Nature $28 \mathrm{Apr}$ 2009 (doi:10.1038/nature07845) 